M. Itô

Nagoya Math. J.

Vol. 57 (1975), 127-152

\title{
SUR L'UNICITÉ DU CÔNE CONVEXE DIVISIBLE CONSTITUÉ PAR DE NOYAUX DE CONVOLUTION DE DIRICHLET
}

\author{
MASAYUKI ITÔ
}

\section{§ 1. Introduction}

Dans toute la suite $\boldsymbol{R}^{n}$ désignera l'espace euclidien à dimension $n$ $(\geqq 1)$. Pour un point $x=\left(x_{1}, x_{2}, \cdots, x_{n}\right)$ de $\boldsymbol{R}^{n}$, on notera $|x|=\left(\sum_{i=1}^{n} x_{i}^{2}\right)^{1 / 2}$. La coordonnée sphérique dans $\boldsymbol{R}^{n}$ s'écrira $(r, \sigma) . \quad \boldsymbol{R}^{+}$désignera l'ensemble $\left\{t \in \boldsymbol{R}^{1} ; t \geqq 0\right\}$.

Rappelons qu'un noyau de convolution $N$ sur $\boldsymbol{R}^{n}$ est une mesure (de Radon) positive dans $\boldsymbol{R}^{n}$ dans la théorie du potentiel. Pour une mesure réelle $\mu$ dans $\boldsymbol{R}^{n}, N * \mu$ s'appelle le $N$-potentiel de $\mu$ dès que cette convolution est définie au sens des mesures. Dans la théorie du potentiel, le noyau de convolution de Hunt posséde les propriétés définitives, qui s'appelle souvent un "bon noyau".

Soit $N_{0}$ un noyau de convolution de Hunt sur $\boldsymbol{R}^{n}$. Dans l'article précédent [7], on définit un cône convexe de Riesz $C_{R}\left(N_{0}\right)$ relatif au noyau $N_{0}$. Cela est, par définition, un cône convexe vaguement fermé de vertex 0 , formé par de noyaux de convolution sur $\boldsymbol{R}^{n}$ et vérifiant les deux conditions suivantes :

(a) $C_{R}\left(N_{0}\right)-\{0\}$ est formé par de noyaux de convolution de Hunt sur $\boldsymbol{R}^{n}$.

(b) $C_{R}\left(N_{0}\right) \ni N_{0}$ et à tout l'élément $N \neq 0$ de $C_{R}\left(N_{0}\right)$, on peut associer un autre élément $N^{\prime} \neq 0$ de $C_{R}\left(N_{0}\right)$ tel que $N * N^{\prime}=N_{0}$.

Dès maintenant on dira qu'un cône convexe de Riesz relatif au noyau $N_{0}$ est un cône convexe divisible relatif au noyau $N_{0}$.

On a obtenu que, dans [6], il existe un seul cône convexe divisible relatif au noyau newtonien $G$ sur $\boldsymbol{R}^{n}(n \geqq 3)$ constitué par de noyaux de convolution sur $\boldsymbol{R}^{n}$ invariants par rotations. Voici un problème si la condition "invariance par rotations" peut être évitée.

Received April 17, 1974. 
Soit $L$ un opérateur différentiel elliptique et auto-adjoint d'ordre $\leqq 2$ à coéfficients constants. On supposera toujours qu'il existe le noyau de convolution $G_{L}$ sur $\boldsymbol{R}^{n}$ s'annulant à l'infini' ${ }^{1)}$ tel que $L G_{L}=-\varepsilon$ (au sens des distributions), où $\varepsilon$ est la mesure de Dirac à l'origine. On désignera par $\left(G_{L, p}\right)_{p \geqq 0}$ la résolvante associée au noyau $G_{L}$.

Le but de cette note est d'énoncer qu'un cône convexe divisible $C_{R}\left(G_{L}\right)$ relatif au noyau $G_{L}$ formé par 0 et de noyaux de convolution de Dirichlet sur $\boldsymbol{R}^{n}$ est uniquement déterminé et que l'on a

$$
\begin{gathered}
C_{R}\left(G_{L}\right)=\left\{c \varepsilon+\int G_{L, p} d \nu(p) ; c \in R^{+} \text {et } \nu \in M^{+}\left(\boldsymbol{R}^{+}\right)\right. \\
\text {avec } \left.\int_{1}^{\infty} \frac{1}{p} d \nu(p)<\infty\right\},
\end{gathered}
$$

où $M^{+}\left(\boldsymbol{R}^{+}\right)$est la totalité des mesures positives sur $\boldsymbol{R}^{+}$.

\section{§ 2. Préliminaires}

Commençons d'abord avec la définition d'un noyau de convolution de Dirichlet sur $\boldsymbol{R}^{n}$. Un noyau de convolution $N$ sur $\boldsymbol{R}^{n}$ est, par définition, un noyau de convolution de Dirichlet si la transformée de Fourier $\hat{N}$ de $N$ est une fonction dans $\boldsymbol{R}^{n}$ et si $1 / \hat{N}$ est une fonction définie-négative et à valeurs réelles (cf. [1]). Une fonction complexe et continue $\psi$ dans $\boldsymbol{R}^{n}$ est, par définition, définie-négative si l'on a: (1) $\psi(0) \geqq 0, \psi(-x)=\overline{\psi(x)}\left(\forall x \in \boldsymbol{R}^{n}\right)$. (2) Quels que soient $m,\left(x^{i}\right)_{i=1}^{m}$ et $\left(\rho_{i}\right)_{i=1}^{m}$ un entier $>0$, une famille de points de $\boldsymbol{R}^{n}$ et une famille de nombres complexes avec $\sum_{i=1}^{m} \rho_{i}=0$, $\sum_{i=1}^{m} \sum_{j=1}^{m} \psi\left(x^{i}-x^{j}\right) \rho_{i} \bar{\rho}_{j} \leqq 0$ (cf. [1]).

On rappelle que, d'après le théorème de Levy-Khinchine, une fonction définie-négative $\psi$ sur $\boldsymbol{R}^{n}$ à valeurs réelles est de la forme

$$
\psi(x)=c+\sum_{i=1}^{m} \sum_{j=1}^{m} a_{i j} x_{i} x_{j}+\int(1-\exp (-2 \pi \sqrt{-1} x \cdot y)) d \mu(y)
$$

pour tout $x=\left(x_{1}, x_{2}, \cdots, x_{n}\right)$ de $\boldsymbol{R}^{n}$, où $c$ est une constante $\geqq 0$, $\sum_{i=1}^{n} \sum_{j=1}^{n} a_{i j} x_{i} x_{j}$ est une forme quadratique symétrique $\geqq 0$ à coéfficients réels, $x \cdot y$ est le produit scalaire de $x$ et $y$ dans $\boldsymbol{R}^{n}$ et où $\mu$ est une mesure positive en dehors de l'origine avec $\int \frac{|x|^{2}}{1+|x|^{2}} d \mu(x)<\infty$ (cf. par exemple, [1]).

1) Cela signifie que, quelle que soit $f$ une fonction finie et continue à support compact, $G_{L * f(x)}$ tend vers 0 avec $|x| \rightarrow \infty$. 
D'après [2], on connaît la remarque suivante:

Remarque 1. Soit $N$ un noyau de convolution sur $\boldsymbol{R}^{n}$. Alors pour que $N$ soit un noyau de convolution de Dirichlet, il faut et il suffit que $N$ soit symétrique par rapport à l'origine et que $N$ soit un noyau de convolution de Hunt.

On rappelle qu'un noyau de convolution de Hunt $N$ sur $\boldsymbol{R}^{n}$ est, par définition, de la forme $N=\int_{0}^{\infty} \alpha_{t} d t$, où $\left(\alpha_{t}\right)_{t \geqq 0}$ est une semi-groupe vaguement continue de mesures positives dans $\boldsymbol{R}^{n}$ avec $\alpha_{0}=\varepsilon$.

Proposition 1. Soit $N$ un noyau de convolution sur $\boldsymbol{R}^{n}$. Alors pour $q u$ 'il existe la résolvante $\left(N_{p}\right)_{p \geqq 0}$ associée au noyau $N$, il faut et il suffit que $N$ soit un noyau de convolution de Hunt sur $\boldsymbol{R}^{n}$ ou bien 0 .

Cela est obtenu dans [5]. Rappelons que $\left(N_{p}\right)_{p \geqq 0}$ est la résolvante associée au noyau $N$ si, pour tous $p \geqq 0, q>0, N_{p}-N_{q}=(q-p) N_{p} * N_{q}$ (Équation résolvante) et si $\lim _{p \downarrow 0} N_{p}=N_{0}=N$ (vaguement).

Pour une fonction définie-négative $\psi$ sur $\boldsymbol{R}^{n}$ à valeurs réelles, il existe une distribution $u$ dans $\boldsymbol{R}^{n}$ symétrique par rapport à l'origine et à croissance lente, et une seule telle que $\hat{u}=-\psi$ (cf. par exemple, [3]).

Proposition 2 (cf. [3]). Soit u une distribution dans $\boldsymbol{R}^{n}$ à croissance lente. Pour que la transformée de Fourier $-\hat{u}$ de $-u$ soit égale à une fonction définie-négative sur $\boldsymbol{R}^{n}$ à valeurs réelles, il faut et il suffit que $u$ soit symétrique par rapport à l'origine et que, quelle que soit $\varphi$ de $C_{K}^{\infty}=C_{K}^{\infty}\left(\boldsymbol{R}^{n}\right), u(\varphi) \leqq 0$ dès que $\varphi$ est réelle et $\varphi(0)=\max _{x \in \boldsymbol{R}^{n}} \varphi(x)$.

On note ici $C_{K}^{\infty}=C_{K}^{\infty}\left(R^{n}\right)$ l'espace de $(L F)$ usuel des fonctions infiniment dérivables dans $\boldsymbol{R}^{n}$ à valeurs complexes et à support compact. Une telle distribution $u$ s'appelle un laplacien généralisé symétrique sur $\boldsymbol{R}^{n}$.

En le généralisant, on dit qu'une distribution $u$ dans $\boldsymbol{R}^{n}$ est conditionnellement positive si, quelle que soit $\varphi$ de $C_{K}^{\infty}, u(\varphi) \geqq 0$ dès que $\varphi \geqq 0$ et le support de $\varphi, \operatorname{supp}(\varphi)$, est contenu en dehors de l'origine. Pour un entier $k \geqq 1, \theta^{k}$ désignera l'idéal de $C_{K}^{\infty}$ formé par de fonctions qui s'annulent ainsi que leurs dérivées d'ordre $<k$ à l'origine. On dit qu'une distribution $u$ dans $\boldsymbol{R}^{n}$ est $k$-conditionnellement positive si, pour toute $\varphi$ de $\theta^{k}, u\left(|\varphi|^{2}\right) \geqq 0$.

Proposition 3 (cf. [3]). Une distribution $u$ dans $\boldsymbol{R}^{n}$ est conditionnellement positive si et seulement s'il existe un entier $k \geqq 1$ tel que $u$ 
soit k-conditionnellement positive.

Cela est un résultat immédiat $d u$ fait que toute la distribution est localement d'ordre fini. Pour discuter une généralisation du théorème de Bochner, on définit une distribution $k$-conditionnellement de type positif, d'après C. S. Herz [3]. Pour un entier $k \geqq 1$, on désigne par $\hat{\theta}^{k}$ l'ensemble des fonctions $\varphi$ de $C_{K}^{\infty}$ telle que $\int x^{\alpha} \varphi(x) d x=0$ pour tout le multi-indice $\alpha=\left(\alpha_{1}, \alpha_{2}, \cdots, \alpha_{n}\right)$ avec $|\alpha|=\sum_{i=1}^{n} \alpha_{i}<k$. On dit qu'une distribution $u$ dans $\boldsymbol{R}^{n}$ est $k$-conditionnellement de type positif si, quelle que soit $\varphi$ de $\hat{\theta}^{k}, u * \varphi * \tilde{\varphi}(0) \geqq 0$, où $\tilde{\varphi}(x)=\overline{\varphi(-x)}$.

Proposition 4 (cf. [3]). Soit $k$ un entier $\geqq 1$. Alors les deux énoncés suivants sont équivalents:

(1) $v$ est une distribution k-conditionnellement de type positif.

(2) $v=\hat{u}$, où $u$ est une distribution $k$-conditionnellement positive dans $\boldsymbol{R}^{n}$ à croissance lente.

On connaît, d'après C.S. Herz [3], que tout l'élément de $\hat{\theta}^{k}$ est de la forme explicite suivante:

Proposition 5. $\hat{\theta}^{k}$ est un idéal de convolution de $C_{K}^{\infty}$ et

$$
\hat{\theta}^{k}=\left\{\sum_{|\alpha|=k} D^{\alpha} g_{\alpha} ; \alpha=\left(\alpha_{1}, \cdots, \alpha_{n}\right): \text { multi-indice, } g_{\alpha} \in C_{K}^{\infty}\right\},
$$

où

$$
D^{\alpha}=\frac{\partial^{|\alpha|}}{\partial x_{1}^{\alpha_{1}} \partial x_{2}^{\alpha_{2}} \cdots \partial x_{n}^{\alpha_{n}}} .
$$

D'après les présentes propositions, on obtient le corollaire suivant:

CoRollaIRE 1. Soient $\psi$ une fonction continue au sens large dans $\boldsymbol{R}^{n}$ et $k$ un entier $\geqq 1$. Si $v=\psi(x) d x$ est une distribution $k$-conditionnellement de type positif dans $\boldsymbol{R}^{n}$, alors $v_{1}=\psi(x, 0, \cdots, 0) d x$ est aussi une distribution k-conditionnellement de type positif dans $\boldsymbol{R}^{1}$ dès que $v_{1}$ définit une distribution dans $\boldsymbol{R}^{1}$.

En effet, $\hat{\theta}^{k}$ étant un idéal de convolution de $C_{K}^{\infty}$, on obtient que, pour toute $\varphi$ de $C_{K}^{\infty}$, la distribution $v_{\varphi}=\psi * \varphi * \tilde{\varphi}(x) d x$ est aussi une distribution $k$-conditionnellement de type positif. Posons $\psi_{1, \varphi}(x)$ $=\psi * \varphi * \tilde{\varphi}(x, 0, \cdots, 0)$ dans $\boldsymbol{R}^{1}$. On a, pour toute $f$ de $C_{K}^{\infty}$, 


$$
0 \leqq v_{\varphi} *\left(\frac{\partial^{k}}{\partial x_{1}^{k}} f\right) *\left(\widetilde{\frac{\partial^{k}}{\partial x_{1}^{k}} f}\right)(0)=(-1)^{k}\left(\frac{\partial^{2 k}}{\partial x_{1}^{2 k}} \psi * \varphi * \tilde{\varphi}\right) * f * \tilde{f}(0)
$$

Donc la fonction $(-1)^{k}\left(\partial^{2 k} / \partial x_{1}^{2 \kappa}\right) \psi * \varphi * \tilde{\varphi}$ est de type positif dans $\boldsymbol{R}^{n}$, et par suite $(-1)^{k}\left(\partial^{2 k} / \partial x^{2 k}\right) \psi_{1, \varphi}$ est aussi une fonction de type positif dans $\boldsymbol{R}^{1}$, car

$$
\frac{\partial^{2 k}}{\partial x_{1}^{2 k}} \psi * \varphi * \tilde{\varphi}(x, 0, \cdots, 0)=\frac{d^{2 k}}{d x^{2 k}} \psi_{1, \varphi}(x)
$$

dans $\boldsymbol{R}^{1}$. La fonction $\varphi$ étant quelconque, la distribution $(-1)^{k}\left(d^{2 k} / d x^{2 k}\right) v_{1}$ est de type positif dans $\boldsymbol{R}^{1}$, et par suite, d'après la proposition 5 , on a, pour toute $f$ de $\hat{\theta}^{k}\left(\boldsymbol{R}^{1}\right), v_{1} * f * \tilde{f}(0) \geqq 0$, d'où $v_{1}$ est $k$-conditionnellement de type positif dans $\boldsymbol{R}^{\mathbf{1}}$.

Rappelons ensuite le théorème de Bernstein (cf. par exemple, [8]).

Proposition 6. Soit $\varphi$ une fonction infiniment dérivable dans $(0,+\infty)$ $\grave{a}$ valeurs réelles. Alors pour que $\varphi$ soit complètement monotone; c'est$\grave{a}$-dire, pour tout l'entier $m \geqq 0,(-1)^{m}\left(d^{m} / d t^{m}\right) \varphi \geqq 0$ dans $(0,+\infty)$, il faut et $i l$ suffit que $\varphi$ soit de la forme $\varphi(t)=\int \exp (-t s) d \nu(s)$ dans $(0,+\infty)$, où $\nu$ est une mesure positive sur $\boldsymbol{R}^{+}$.

Dans ce cas, $\nu$ est uniquement déterminée, d'après l'injectivité de la transformation de Laplace. D'après la proposition 6, on obtient le corollaire suivant:

CoRollaIRE 2. Soient a une constante $\geqq 0$ et $\kappa$ une mesure positive sur $\boldsymbol{R}^{+}$. Alors pour que, pour tout l'entier $m \geqq 0,\{-(d / d t-a)\}^{m} \kappa \geqq 0$ au sens des distributions dans $(0,+\infty)$, où $\{-(d / d t)-a\}^{0} \kappa=\kappa$, il faut et $i l$ suffit que $\kappa$ soit de la forme

$$
\kappa=c \varepsilon+\left(\int_{a}^{\infty} \exp (-p t) d \nu(t)\right) d t
$$

où c est une constante $\geqq 0$ et où $\nu$ est une mesure positive sur $\boldsymbol{R}^{+}$portée $\operatorname{par}[a,+\infty)$.

En effet, la condition est évidemment suffisante. On montrera son inverse. Il existe une constante $c>0$ et une fonction infiniment dérivable $\varphi \geqq 0$ dans $(0,+\infty)$ avec $\int_{0}^{1} \varphi(t) d t<+\infty$ telles que $\kappa=c \varepsilon+\varphi(t) d t$. On a, pour tout l'entier $m \geqq 0,\{-(d / d t)-a\}^{m} \varphi(t) \geqq 0$ dans $(0,+\infty)$. Il est 
facilement montré, par récurrence pour $k$, que pour tous les entier $m \geqq 0, k \geqq 0$ et pour tout $b$ avec $0 \leqq b \leqq a,\{-(d / d t)-b\}^{k}\{-(d / d t)-a\}^{m} \varphi(t)$ $\geqq 0$ dans $(0,+\infty)$, d'après

$$
\begin{aligned}
\left(-\frac{d}{d t}-b\right)^{k+1}\left(-\frac{d}{d t}-a\right)^{m} \varphi= & \left(-\frac{d}{d t}-b\right)^{k}\left(-\frac{d}{d t}-a\right)^{m+1} \varphi \\
& +(a-b)\left(-\frac{d}{d t}-b\right)^{k}\left(-\frac{d}{d t}-a\right)^{m} \varphi
\end{aligned}
$$

Donc, d'après la proposition 6 , il existe une mesure positive $\nu$ dans $\boldsymbol{R}^{+}$ telle que l'on ait $\varphi(t)=\int \exp (-p t) d \nu(p)$ dans $(0,+\infty)$. On a ensuite $\{-(d / d t)-a\} \varphi(t)=\int \exp (-p t)(p-a) d \nu(p)$, et donc $(p-a) d \nu(p)$ doit être une mesure positive sur $\boldsymbol{R}^{+}$, d'où $\nu$ est portée par $[a,+\infty)$.

Dans ce cas, $c$ et $\nu$ sont aussi uniquement déterminées.

\section{§3. Notre théorème principal}

On préparera d'abord quelque lemmes suivants.

LEMME 1. Soit $\psi$ une fonction définie-négative dans $\boldsymbol{R}^{1} \grave{a}$ valeurs réelles. Pour que, pour tout l'entier $m \geqq 0,(-1)^{m+1}|t|^{2 m} \psi(t) d t$ soit conditionnellement de type positif dans $\boldsymbol{R}^{1}$, il faut et il suffit que

$$
\psi(t)=c_{1}+c_{2} t^{2}+\int\left(1-\frac{p}{p+4 \pi^{2} t^{2}}\right) d \alpha(p),
$$

où $c_{i}(i=1,2)$ est une constante $\geqq 0$ et où $\alpha$ est une mesure positive dans $(0,+\infty)$.

De la même manière que ci-dessous et d'après le corollaire 2 , le lemme suivant a lieu.

Lemme 1'. Soient $\psi$ la même que ci-dessus et a une constante $\geqq 0$. Alors pour que, pour tout l'entier $m \geqq 0,(-1)^{m+1}\left(|t|^{2}+a\right)^{m} \psi(t) d t$ soit conditionnellement de type positif, il faut et $i l$ suffit que $\psi$ soit de la même forme que dans le lemme 1 , où $c_{1} \geqq c_{2} \sqrt{a}$ et $\nu$ est portée par $[\sqrt{a}, \infty)$.

En général, on dit qu'une distribution $v$ dans $\boldsymbol{R}^{n}$ est conditionnellement de type positif s'il existe un entier $k \geqq 1$ telle que $v$ soit $k$-conditionnellement de type positif. 
Démonstration du lemme 1. Montrons d'abord que la condition est nécessaire. D'après le théorème de Levy-Khinchine, $\psi$ est de la forme

$$
\psi(t)=c_{1}+c_{2} t^{2}+\int(1-\exp (-2 \pi \sqrt{-1} t s)) d \mu(s)
$$

dans $\boldsymbol{R}^{1}$, où $c_{i}(i=1,2)$ est une constante $\geqq 0$ et où $\mu$ est une mesure positive en dehors de l'origine symétrique par rapport à l'origine avec $\int \frac{|t|^{2}}{1+|t|^{2}} d \mu(t)<+\infty$. Soit $u$ le laplacien généralisé symétrique sur $\boldsymbol{R}^{1}$ tel que $\hat{u}=-\psi$. Alors $u=\mu$ en dehors de l'origine. D'après notre hypothèse et la proposition 4 , pour tout l'entier $m \geqq 0$, la distribution $\left(d^{2 m} / d t^{2 m}\right) u$ est conditionnellement positive dans $\boldsymbol{R}^{1}$, et par suite $d^{2 m} u / d t^{2 m} \geqq 0$ au sens des distributions en dehors de l'origine. Donc il existe une fonction infiniment dérivable $\varphi$ dans $(0,+\infty)$ telle que $\mu=\varphi(|t|) d t$ en dehors de l'origine et pour tout l'entier $m \geqq 0,\left(d^{2 m} / d t^{2 m}\right) \varphi(t) \geqq 0$ dans $(0,+\infty)$. D'après $\int_{1}^{\infty} \varphi(t) d t=\int_{1}^{\infty} d \mu<+\infty$ et $\frac{d^{2}}{d t^{2}} \varphi \geqq 0$ dans $(0,+\infty)$, on a $(d / d t) \varphi \leqq 0$ dans $(0,+\infty)$, et par suite $\lim _{t \rightarrow+\infty} \varphi(t)=0$. Donc, pour toute $f \geqq 0$ de $C_{K}^{\infty}\left(\boldsymbol{R}^{1}\right)$ et pour tout l'entier $m \geqq 0$,

$$
\lim _{t \rightarrow+\infty} \int\left(\frac{d^{2 m}}{d t^{2 m}} \varphi\right)(t-s) f(s) d s=\lim _{t \rightarrow+\infty} \int \varphi(t-s)\left(\frac{d^{2 m}}{d t^{2 m}} f\right)(s) d s=0 .
$$

D'après cette égalité et $\left(d^{2(m+1)} / d t^{2(m+1)}\right) \varphi \geqq 0$ dans $(0,+\infty)$, on a $\left(d^{2 m+1} / d t^{2 m+1}\right) \varphi \leqq 0$ dans $(0,+\infty)$. En utilisant la proposition 6 , il existe une mesure positive $\nu$ sur $\boldsymbol{R}^{+}$telle que l'on ait

$$
\varphi(t)=\int \exp (-t s) d \nu(s) \quad \text { dans } \quad(0,+\infty)
$$

D'après $\lim _{t \rightarrow+\infty} \varphi(t)=0$, on a $\nu(\{0\})=0$. Par conséquent, on a

$$
\begin{aligned}
\varphi(t)= & c_{1}+c_{2} t^{2}+\iint(1-\exp (-2 \pi \sqrt{-1} t s)) \int \exp (-|s| p) d \nu(p) d s \\
= & c_{1}+c_{2} t^{2}+\iint(1-\exp (-2 \pi \sqrt{-1} t s)) \exp (-p|s|) d s d \nu(p) \\
= & c_{1}+c_{2} t^{2}+\int\left(\int_{0}^{\infty}(1-\exp (-2 \pi \sqrt{-1} t s)) \exp (-p s) d s\right. \\
& \left.+\int_{-\infty}^{0}(1-\exp (2 \pi \sqrt{-1} t s)) \exp (p s) d s\right) d \nu(p)
\end{aligned}
$$




$$
\begin{aligned}
& =c_{1}+c_{2} t^{2}+\int\left(\frac{2}{p}-\frac{2 p}{p^{2}+4 \pi^{2} t^{2}}\right) d \nu(p) \\
& =c_{1}+c_{2} t^{2}+\int\left(1-\frac{p}{p+4 \pi^{2} t^{2}}\right) d \alpha(p)
\end{aligned}
$$

où $\alpha$ est une mesure positive dans $(0,+\infty)$ définie par $\int f d \alpha$ $=\int f\left(t^{2}\right) \frac{2}{t} d \nu(t)$ pour toute la fonction finie et continue $f$ dans $(0,+\infty)$ à support compact.

Supposons réciproquement que, avec deux constantes $c_{1} \geqq 0, c_{2} \geqq 0$ et une mesure positive $\alpha$ dans $(0,+\infty), \psi(t)=c_{1}+c_{2} t^{2}+\int\left(1-\frac{p}{p+4 \pi^{2} t^{2}}\right) d \alpha(p)$. Evidemment $\left(c_{1}+c_{2} t^{2}+\int_{1 / m}^{m}\left(1-\frac{p}{p+4 \pi^{2} t^{2}}\right) d \alpha(p)\right) d t$ converge vers $\psi(t) d t$ au sens des distributions dans $\boldsymbol{R}^{1}$ avec $m \rightarrow+\infty$. Donc on peut supposer que $\alpha$ est à support compact, et par suite

$$
\psi(t)=c_{0}+c_{2} t^{2}-\int \frac{p}{p+4 \pi^{2} t^{2}} d \alpha(p),
$$

où $c_{0}=c_{1}+\int d \alpha$. Donc il est évident que la distribution $-\psi d t$ est 1-conditionnellement de type posititif dans $\boldsymbol{R}^{1}$. Soit $u$ le laplacien généralisé symétrique sur $\boldsymbol{R}^{1}$ tel que $\hat{\mathrm{u}}=-\psi$. On a, pour tout l'entier $m \geqq 1$,

$$
\begin{aligned}
\widehat{\frac{d^{2 m}}{d t^{2 m}} u(t)=} & (-1)^{m+1}\left(4 \pi^{2} t^{2}\right)^{m} \psi(t) \\
= & (-1)^{m+1}\left(4 \pi^{2} t^{2}\right)^{m}\left(c_{0}+c_{2} t^{2}\right)+\sum_{i=1}^{m}(-1)^{m+1-i}\left(4 \pi^{2} t^{2}\right)^{m-i} \int p^{i} d \alpha(p) \\
& +\int \frac{p^{m+1}}{p+4 \pi^{2} t^{2}} d \alpha(p),
\end{aligned}
$$

et donc, avec le noyau de convolution $G_{p}$ sur $R^{1}$ tel que $\widehat{G p}=1 /\left(p+4 \pi^{2} t^{2}\right)$ $(\forall p>0)$,

$$
\begin{aligned}
\frac{d^{2 m}}{d t^{2 m}} u= & c_{2} \frac{d^{2(m+1)}}{d t^{2(m+1)}} \varepsilon-c_{0} \frac{d^{2 m}}{d t^{2 m}} \varepsilon-\sum_{i=1}^{m}\left(\int p^{i} d \alpha(p)\right) \frac{d^{2(m-i)}}{d t^{2(m-i)}} \varepsilon \\
& +\int p^{m+1} G_{p} d \alpha(p)
\end{aligned}
$$


Par conséquent, $\left(d^{2 m} / d t^{2 m}\right) u$ est conditionnellement positive (précisément, $(m+1)$-conditionnellement positive) dans $\boldsymbol{R}^{1}$. D'après la proposition 4 , la distribution $(-1)^{m+1} t^{2 m} \psi(t) d t$ est conditionnellement de type positif dans $\boldsymbol{R}^{1}$. La démonstration est ainsi complète.

Le lemme suivant résultera du lemme 1 et du corollaire 1 . On désigne par $S_{1,0}$ la sphère d'unité de centre 0 et par $M^{+}((0,+\infty))$ la totalité des mesures positives dans $(0,+\infty)$.

LEMME 2. Soit $\psi$ une fonction définie-négative dans $\boldsymbol{R}^{n}(n \geqq 1)$ $\grave{a}$ valeurs réelles. Si, pour tout l'entier $m \geqq 0$, la distribution $(-1)^{m+1}|x|^{2 m} \psi(x) d x$ dans $\boldsymbol{R}^{n}$ est conditionnellement de type positif, alors il existe une constante $c \geqq 0$, une fonction non-négative, finie et continue $q(\sigma)$ sur $S_{1,0}$ et une famille $\left(\alpha_{\sigma}\right)_{\sigma \in S_{1,0}}$ dans $M^{+}((0,+\infty))$ telles que

$$
\psi(x)=\psi(r, \sigma)=c+q(\sigma) r^{2}+\int\left(1-\frac{p}{p+4 \pi^{2} r^{2}}\right) d \alpha_{\sigma}(p) .
$$

En effet, pour tout $\sigma$ de $S_{1,0}, \psi_{\sigma}$ désigne la fonction obtenue de $\psi$ par la rotation de $\sigma$. Alors $\psi_{\sigma}$ est aussi définie-négative et à valeurs réelles. Pour tout l'entier $k \geqq 1, \hat{\theta}^{k}$ étant invariant par rotations, la distribution $(-1)^{m+1}|x|^{2 m} \psi_{\sigma}(x) d x$ dans $\boldsymbol{R}^{n}$ est aussi conditionnellement de type positif. Posons $\psi_{\sigma, 1}(t)=\psi_{\sigma}(t, 0, \cdots, 0)$ sur $\boldsymbol{R}^{1}$; alors $\psi_{\sigma, 1}$ est définienégative dans $\boldsymbol{R}^{1}$. D'après le corollaire 1 , pour tout l'entier $m \geqq 0$, $(-1)^{m+1} t^{2 m} \psi_{\sigma, 1}(t) d t$ est une distribution conditionnellement de type positif dans $\boldsymbol{R}^{1}$, et alors, d'après le présent lemme 1 , il existe deux constantes $c_{1, \sigma} \geqq 0, c_{2, \sigma} \geqq 0$ et une mesure positive $\alpha_{\sigma}^{\prime}$ dans $(0,+\infty)$ telles que

$$
\psi_{\sigma, 1}(t)=c_{1, \sigma}+c_{2, \sigma} t^{2}+\int\left(1-\frac{p}{p+4 \pi^{2} r^{2}}\right) d \alpha_{\sigma}^{\prime}(p) .
$$

D'après $\psi_{\sigma, 1}(0)=\psi(0), c_{1, \sigma}$ ne dépend pas de $\sigma$, et donc on pose $c=c_{1, \sigma}$. Par conséquent, il existe une fonction non-négative $q(\sigma)$ sur $S_{1,0}$ et une famille $\left(\alpha_{\sigma}\right)_{\sigma \in S_{1,0}}$ dans $M^{+}((0,+\infty))$ telles que

$$
\psi(r, \sigma)=c+q(\sigma) r^{2}+\int\left(1-\frac{p}{p+4 \pi^{2} r^{2}}\right) d \alpha_{\sigma}(p) .
$$

Rappelons le théorème de Levy-Khinchine et le lemme 1; alors on a, pour tout $\sigma$ de $S_{1,0}$,

$$
\lim _{r \rightarrow \infty} \frac{1}{r^{2}} \int\left(1-\frac{p}{p+4 \pi^{2} r^{2}}\right) d \alpha_{\sigma}(p)=0
$$


et donc, en utilisant encore le théorème de Levy-Khinchine, il existe une mesure positive $\mu$ dans $\boldsymbol{R}^{n}-\{0\}$ telle que

$$
\begin{gathered}
\int \frac{|x|^{2}}{1+|x|^{2}} d \mu(x)<\infty \text { et } \int(1-\exp (-2 \pi \sqrt{-1} x \cdot y)) d \mu(y) \\
=\int\left(1-\frac{p}{p+4 \pi^{2} r^{2}}\right) d \alpha_{o}(p)
\end{gathered}
$$

où $x=(r, \sigma)$. Donc la fonction $\int\left(1-\frac{p}{p+4 \pi^{2} r^{2}}\right) d \alpha_{\sigma}(p)$ de $(r, \sigma)$ est finie et continue dans $\boldsymbol{R}^{n}$, et par suite la continuité de $q$ en résulte.

LEMME 3. Soient $L$ un opérateur différentiel elliptique et auto-adjoint d'ordre $\leqq 2$ à coéfficients constants et $G_{L}$ le noyau de convolution sur $\boldsymbol{R}^{n}$ s'annulant à l'infini tel que $E G_{L}=-\varepsilon_{.}{ }^{2)}$ Soit $N$ un noyau de convolution $\neq 0$ sur $\boldsymbol{R}^{n}$ symétrique par rapport à l'origine et s'annulant à l'infini. Alors pour que la distribution $L N$ soit conditionnellement positive, il faut et il suffit qu'il existe un noyau de convolution de Dirichlet $N_{1}$ sur $\boldsymbol{R}^{n}$ tel que $N * N_{1}=G_{L}$. Dans ce cas, $N_{1}$ est uniquement déterminé.

Remarque 2. Soient $k$ un entier avec $1 \leqq k \leqq n$ et $L=\sum_{i=1}^{k} \frac{\partial^{2}}{\partial^{2} x_{i}}-a$; supposons qu'il existe un noyau de convolution $G_{L}$ sur $\boldsymbol{R}^{n}$ s'annulant à l'infini tel que $L G_{L}=-\varepsilon$. Soit ensuite $N$ un noyau de convolution sur $\boldsymbol{R}^{n}$ s'annulant à l'infini. Si $L N$ est conditionnellement positive, alors $\operatorname{supp}(N) \subset\left\{x=\left(x_{1}, x_{2}, \cdots, x_{n}\right) \in \boldsymbol{R}^{n} ; x_{k+1}=\cdots=x_{n}=0\right\}$.

En effet, on connaît bien $\operatorname{supp}\left(G_{L}\right) \subset\left\{x=\left(x_{1}, \cdots, x_{k}, 0, \cdots, 0\right) \in \boldsymbol{R}^{n}\right\}$. Pour une fonction finite et continue $f \geqq 0$ dans $\boldsymbol{R}^{n}$ à support compact, $G_{L} * f(x)>0$ dans $\left\{x \in \boldsymbol{R}^{n} ; f(x)>0\right\}$, car $\operatorname{supp}\left(G_{L}\right) \ni 0$. Donc, pour toute la fonction finie et continue $g \geqq 0$ dans $\boldsymbol{R}^{n}$ à support contenu dans $\left\{x \in \boldsymbol{R}^{n} ; f(x)>0\right\}$, il existe une constante $a_{g}>0$ telle que $N * g(x) \leqq a_{g} G_{L} * f(x)$ sur $\operatorname{supp}(g) . \quad N * g$ étant $L$-sous-harmonique en dehors de $\operatorname{supp}(g)$ et ayant $\lim _{|x| \rightarrow \infty} N * g(x)=0, N * g(x) \leqq a_{g} G_{L} * f(x)$ dans $\boldsymbol{R}^{n}$. La fonction $g$ étant quelconque, on a $\operatorname{supp}(N * f) \subset \operatorname{supp}\left(G_{L} * f\right)$, d'où $\operatorname{supp}(N) \subset$ $\operatorname{supp}\left(G_{L}\right)$.

Montrons le lemme 3. S'il existe un noyau de convolution de Dirichlet $N_{1}$ sur $\boldsymbol{R}^{n}$ tel que $N * N_{1}=G_{L}$, alors $(L N) * N_{1}=-\varepsilon$, et par suite $L N$ est un laplacien généralisé symétrique sur $\boldsymbol{R}^{n}$, d'où la condition est suffisante.

\footnotetext{
2) Il est bien connu que $G_{L}$ est uniquement déterminé.
} 
On obtient, en même temps, l'unicité de $N_{1}$. Supposons réciproquement que $L N$ est conditionnellement positive. Si $L$ est constant, alors notre énoncé est évident. Donc, d'après la remarque 2 et en utilisant une certaine transformation linéaire de $\boldsymbol{R}^{n}$ à lui-même on peut supposer que $L$ est uniformément elliptique. $N$ est absolument continu (par rapport à la mesure de Lebesgue) en dehors de l'origine. Rapplons le lemme suivant, qui est obtenu pour un noyau d'un cadre plus large dans [4].

LEMME 4. Soit $N$ un noyau de convolution sur $\boldsymbol{R}^{n}$ symétrique par rapport à l'origine et absolument continu en dehors de l'origine. Alors, pour une constante $c>0$, une fonction non-négative, mesurable et localement bornée $u$ dans $\boldsymbol{R}^{n}$ et pour un compact $K$ dans $\boldsymbol{R}^{n}$, il existe une fonction non-négative, mesurable et bornée $f_{c, u, K}$ dans $\boldsymbol{R}^{n}$ portée par $K$ telle que l'on ait $N f_{c, u, K}+c f_{c, u, K} \geqq u$ presque partout (noté p.p.) sur $K$ et $N f_{c, u, K}+c f_{c, u, K}=u$ p.p. sur $\left\{x \in \boldsymbol{R}^{n} ; f_{c, u, K}(x)>0\right\}$.

On note ici $N f_{c, u, K}$ la densité de la mesure $N *\left(f_{c, u, K} d x\right)$ par rapport à la mesure de Lebesgue.

Continuons la démonstration du lemme 3. Soient $\varphi$ une fonction non-négative, finie et continue dans $\boldsymbol{R}^{n}$ à support compact telle que $\int \varphi d x=1$ et $B_{m, 0}$ la boule ouverte de centre 0 et de rayon $m$; posons $\varphi_{m}(x)=m^{n} \varphi(m x)$. Pour trois entiers $j>0, k>0, m>0$ et pour un nombre $p>0$, on désigne par $f_{j, k, m, p}$ la fonction obtenue dans le lemme 4 pour le noyau de convolution $N+p G_{L}$ sur $R^{n}, c=1 / k, u=G_{L} * \varphi_{m}$ et pour $K=\overline{B_{j, 0}}$. On peut écrire $N=a \varepsilon+K(x) d x$, où $a$ et $K$ sont respectivement une constante non-négative et une fonction non-négative et localement sommable dans $\boldsymbol{R}^{n}$. On a

$$
K * f_{j, k, m, p} \leqq G_{L} * \varphi_{m}-p G_{L} * f_{j, k, m, p} \text { p.p. } \operatorname{sur}\left\{x \in \boldsymbol{R}^{n} ; f_{j, k, m, p}(x)>0\right\} .
$$

D'après la continuité de $K * f_{j, k, m, p}$, la présente inégalité a lieu partout sur le support $\operatorname{supp}\left(f_{j, k, m, p} d x\right)$. Ayant $\lim _{|x| \rightarrow \infty} K * f_{j, k, m, p}(x)=0$ et $K * f_{j, k, m, p}$ étant $L$-sous-harmonique en dehors de $\operatorname{supp}\left(f_{j, k, m, p} d x\right)$, on a

$$
K * f_{j, k, m, p} \leqq G_{L} * \varphi_{m}-p G_{L} * f_{j, k, m, p} \quad \text { sur } \quad \boldsymbol{R}^{n},
$$

et par suite

$$
\left(N+p G_{L}\right) f_{j, k, m, p}+\frac{1}{k} f_{j, k, m, p} \leqq G_{L} * \varphi_{m} \quad p . p . \text { sur } \quad R^{n},
$$




$$
\left(N+p G_{L}\right) f_{j, k, m, p}+\frac{1}{k} f_{j, k, m, p}=G_{L} * \varphi_{m} \quad p . p . \operatorname{sur} \quad \overline{B_{j, 0}} .
$$

En faisant $m \rightarrow+\infty$ et ensuite $k \rightarrow+\infty$, on obtient qu'il existe une mesure positive $\mu_{j, p}$ dans $\boldsymbol{R}^{n}$ portée par $\overline{B_{j, 0}}$ telle que, au sens des mesures,

$$
\begin{array}{lll}
\left(N+p G_{L}\right) * \mu_{j, p} \leqq G_{L} & \text { dans } & \boldsymbol{R}^{n}, \\
\left(N+p G_{L}\right) * \mu_{j, p}=G_{L} & \text { dans } & B_{j, 0} .
\end{array}
$$

D'après $p G_{L} * \mu_{j, p} \leqq G_{L}$, on a $p \int d \mu_{j, p} \leqq 1$. Soit $N_{p}^{(1)}$ un point vaguement adhérent de $\left(\mu_{j, p}\right)_{j=1}^{\infty}$ lorsque $j \rightarrow \infty$; alors, d'après l'hypothèse que $N+p G_{L}$ s'annule à l'infini, on a

$$
\left(N+p G_{L}\right) * N_{p}^{(1)}=G_{L} \quad \text { dans } \quad R^{n} .
$$

On a aussi $p \int d N_{p}^{(1)} \leqq 1$. On obtient ainsi une famille $\left(N_{p}^{(1)}\right)_{p>0}$ de noyaux de convolution sur $\boldsymbol{R}^{n}$ symétriques par rapport à l'origine. On a, pour tous $p>0$ et $q>0$,

$$
\begin{aligned}
G_{L} * N_{p}^{(1)}= & \left(N+q G_{L}\right) * N_{q}^{(1)} * N_{p}^{(1)}=\left(N+p G_{L}\right) * N_{p}^{(1)} * N_{q}^{(1)} \\
& +(q-p) G_{L} * N_{p}^{(1)} * N_{q}^{(1)}=G_{L} * N_{q}^{(1)}+(q-p) G_{L} * N_{p}^{(1)} * N_{q}^{(1)},
\end{aligned}
$$

et donc

$$
N_{p}^{(1)}=N_{q}^{(1)}+(q-p) N_{p}^{(1)} * N_{q}^{(1)} .
$$

Ayant $N \neq 0$ et $\left(N_{p}^{(1)}\right)_{p>0}$ étant croissante avec $p \downarrow 0$, on obtient que $N_{1}$ $=\lim _{p \downarrow 0} N_{p}^{(1)}$ définit un noyau de convolution sur $\boldsymbol{R}^{n}$ symétrique par rapport à l'origine et que $\left(N_{p}^{(1)}\right)_{p>0}$ est la résolvante associée au noyau $N_{1}$, où $N_{0}^{(1)}=N_{1}$. D'après $N_{1} \neq 0, N_{1}$ est un noyau de convolution de Dirichlet sur $\boldsymbol{R}^{n}$ (cf. la remarque 1 et la proposition 1). On a évidemment $N * N_{1} \leqq G_{L}$ dans $\boldsymbol{R}^{n}$. Soient $m$ un entier positif et $\nu_{m}$ la mesure positive dans $\boldsymbol{R}^{n}$ portée par $S_{m, 0}$ telle que $G_{L} * \nu_{m}=G_{L}$ sur $C B_{m, 0}$. Alors la famille $\left(N * N_{p}^{(1)} *\left(\varepsilon-\nu_{m}\right)\right)_{p>0}$ converge vaguement vers $G_{L} *\left(\varepsilon-\nu_{m}\right)$ dans $\boldsymbol{R}^{n}$ avec $p \rightarrow 0$. Ayant $\operatorname{supp}\left(N *(\varepsilon-\nu)^{+}\right) \subset \overline{B_{m, 0}}$, on obtient

$$
G_{L} \geqq N * N_{1} \geqq N * N_{1} *\left(\varepsilon-\nu_{m}\right) \geqq G_{L} *\left(\varepsilon-\nu_{m}\right)
$$

dans $\boldsymbol{R}^{n}$. En faisant $m \rightarrow \infty$, on arrive à l'égalité $N * N_{1}=G_{L}$, d'où la condition est nécessaire.

On note $D_{L}\left(\boldsymbol{R}^{n}\right)$ l'ensemble formé par tous les noyaux de convolution de Dirichlet $N$ sur $\boldsymbol{R}^{n}$ tels que $L N$ soit conditionnellement positive. 
Corollaire 3. $\grave{A}$ tout l'élément $N$ de $D_{L}\left(\boldsymbol{R}^{n}\right)$, on peut associer uniquement un autre élément $N^{\prime}$ de $D_{L}\left(\boldsymbol{R}^{n}\right)$ tel que $N * N^{\prime}=G_{L}$.

De la même manière que pour le laplacien ordinaire (cf. [6]), on dit que, pour un opérateur elliptique différentiel et auto-adjoint $L$ d'ordre $\leqq 2$ à coéfficients constants, un noyau de convolution $N$ sur $\boldsymbol{R}^{n}$ est complètement $L$-sous-harmonique si, pour tout l'entier $m \geqq 0, L^{m} N$ est conditionnellement positive, où $L^{0} N=N, L^{1}=L$ et $L^{m}=L^{m-1} L\left({ }^{\forall} m \geqq 2\right)$.

LEMme 5. Soient $N$ un noyau de convolution sur $\boldsymbol{R}^{n}$ symétrique par rapport à l'origine et s'annulant à l'infini, et a une constante $\geqq 0$. On suppose que si $n=1,2$, alors $a>0$. Si $N$ est complètement $(\Delta-a)$ sous-harmonique, où $\Delta$ est le laplacien ordinaire sur $\boldsymbol{R}^{n}$, alors il existe une constante $c \geqq 0$ et une famille $\left(\alpha_{\sigma}\right)_{\sigma \in S_{1,0}}$ dans $M^{+}\left(\boldsymbol{R}^{+}\right)$telles que

$$
\hat{N}(x)=\hat{N}(r, \sigma)=c+\int \frac{1}{p+4 \pi^{2} r^{2}} d \alpha_{\sigma}(p) .
$$

Dans ce cas, la famille $\left(\alpha_{\sigma}\right)_{\sigma \in S_{1,0}}$ est unique et, pour tout $\sigma$ de $S_{1,0}$, $\operatorname{supp}\left(\alpha_{\sigma}\right) \subset[a, \infty)$.

En effet, soient $b$ une constante avec $0 \leqq b \leqq a$ et $k$ un entier $\geqq 0$. Si, pour tout l'entier $m \geqq 0,(\Delta-a)^{m}(\Delta-b)^{k} N$ est conditionnellement positive, alors, d'après

$$
(\Delta-a)^{m}(\Delta-b)^{k+1} N=(a-b)(\Delta-a)^{m}(\Delta-b)^{k} N+(\Delta-a)^{m+1}(\Delta-b)^{k} N,
$$

$(\Delta-a)^{m}(\Delta-b)^{k+1} N$ l'est aussi. Donc, pour tous les entiers $m \geqq 0, k \geqq 0$ et pour tout $b$ avec $0 \leqq b \leqq a,(\Delta-a)^{m}(\Delta-b)^{k} N$ est aussi conditionnellement positive. Soit $b$ une constante telle que $0 \leqq b \leqq a$ ou bien $0<b \leqq a$ d'accord avec $n \geqq 3$ ou bien $n=1,2$. D'après l'existence du noyau de convolution s'annulant à l'infini $G_{b}$ sur $\boldsymbol{R}^{n}$ avec $(\Delta-b) G_{b}=-\varepsilon$ et le lemme 3 , il existe une fonction définie-négative $\psi_{b}$ dans $\boldsymbol{R}^{n}$ telle que $\hat{N}(x)=\psi_{b}(x) /\left(4 \pi^{2}|x|^{2}+b\right)$. Pour $n=1,2$, en faisant $b \rightarrow 0$, on obtient aussi qu'il existe une fonction définie-négative $\psi_{0}$ dans $\boldsymbol{R}^{n}$ à valeurs réelles telle que $\hat{N}(x)=\psi_{0}(x) / 4 \pi^{2}|x|^{2}$. D'après la proposition 4 , pour tout l'entier $m \geqq 0,(-1)^{m}|x|^{2 m} \hat{N}(x) d x$ est conditionnellement de type positif, et par suite $(-1)^{m+1}|x|^{2 m} \psi_{0}(x) d x$ l'est aussi. D'après le lemme 2 , il existe une constante $c_{1} \geqq 0$, une fonction finie et continue $q(\sigma) \geqq 0$ sur $S_{1,0}$ et une famille $\left(\alpha_{\sigma}^{\prime}\right)_{\sigma \in S_{1,0}}$ dans $M^{+}(0, \infty)$ telles que 


$$
\psi_{0}(x)=\psi(r, \sigma)=c_{1}+q(\sigma) r^{2}+\int\left(1-\frac{p}{p+4 \pi^{2} r^{2}}\right) d \alpha_{\sigma}^{\prime}(p)
$$

La fonction $\psi_{0}(x) /|x|^{2}$ étant de type positif, pour tout l'entier $m \geqq 0$, $\psi_{0}(m x) / m^{2}|x|^{2}$ l'est aussi. Faisant $m \rightarrow \infty$, on obtient que la fonction $q(\sigma)$ de $(r, \sigma)$ est de type positif, d'où $q(\sigma)$ est constante $\geqq 0$. Posons $c=q(\sigma) / 4 \pi^{2}$ et $\alpha_{\sigma}=c_{1} \varepsilon+\alpha_{\sigma}^{\prime}$; alors $\alpha_{\sigma} \in M^{+}\left(\boldsymbol{R}^{+}\right)$et

$$
\hat{N}(x)=\hat{N}(r, \sigma)=c+\int \frac{1}{p+4 \pi^{2} r^{2}} d \alpha_{\sigma}(p) .
$$

Voyons l'unicité de $\left(\alpha_{\sigma}\right)_{\sigma \in S_{1,0}}$. Soient $c^{\prime}$ une constante $\geqq 0$ et $\left(\alpha_{\sigma}^{\prime}\right)_{\sigma \in S_{1,0}}$ une famille de $M^{+}\left(\boldsymbol{R}^{+}\right)$telles que $\hat{N}(r, \sigma)=c^{\prime}+\int \frac{1}{p+4 \pi^{2} r^{2}} d \alpha_{\sigma}^{\prime}(p)$. En faisant $r \rightarrow+\infty$, on obtient $c=c^{\prime}$. Ayant, pour tout $\sigma$ de $S_{1,0}$,

$$
\int \frac{1}{p+4 \pi^{2} r^{2}} d \alpha_{\sigma}(p)=\int_{0}^{\infty} \int \exp \left(-4 \pi^{2} r^{2} t\right) \exp (-p t) d \alpha_{\sigma}(p) d t
$$

et

$$
\begin{aligned}
\int_{0}^{\infty} \int \exp \left(-4 \pi^{2} r^{2} t\right) \exp (-p t) d \alpha_{\sigma}(p) d t \\
=\int_{0}^{\infty} \int \exp \left(-4 \pi^{2} r^{2} t\right) \exp (-p t) d \alpha_{\sigma}^{\prime}(p) d t,
\end{aligned}
$$

on obtient, d'après l'injectivité de la transformation de Laplace,

$$
\int \exp (-p t) d \alpha_{\sigma}(p)=\int \exp (-p t) d \alpha_{\sigma}^{\prime}(p)
$$

dans $(0, \infty)$, et encore, de la même manière, $\alpha_{\sigma}=\alpha_{\sigma}^{\prime}$.

Voyons finalement $\operatorname{supp}\left(\alpha_{\sigma}\right) \subset[a, \infty)$. Pour tout l'entier $m \geqq 0$, la distribution $(-1)^{m+1}|x|^{2 m} \psi_{a}(x) d x$ étant conditionnellement de type positif et ayant

$$
\begin{aligned}
\psi_{a}(r, \sigma) & =\left(4 \pi^{2} r^{2}+a\right) \hat{N}(r, \sigma) \\
& =c\left(4 \pi^{2} r^{2}+a\right)+\int\left(1-\frac{p-a}{p+4 \pi^{2} r^{2}}\right) d \alpha_{\sigma}(p),
\end{aligned}
$$

on obtient, d'après le lemme 2 et la présente unicité, $(p-a) d \alpha_{\sigma}(p)$ est une mesure positive, d'où $\operatorname{supp}\left(\alpha_{\sigma}\right) \subset[a, \infty)$.

D'après les présents lemmes, on obtient la proposition suivante:

Proposition 7. Soit $L$ un opérateur différentiel elliptique d'ordre 
$\leqq 2$ à coéfficients constants et supposons qu'il existe le noyau de convolution $G_{L}$ sur $\boldsymbol{R}^{n}$ s'annulant à l'infini avec $L G_{L}=-\varepsilon$. Soit $N$ un noyau de convolution sur $\boldsymbol{R}^{n}$ symétrique par rapport à l'origine et s'annulant à l'infini. Alors pour que $N$ soit complètement L-sous-harmonique et que, pour tout l'entier $m \geqq 0$, il existe une constante $c_{m}>0$ telle que $L^{m} N$ $\leqq c_{m} G_{L}$ au sens des distributions en dehors de l'origine, il faut et il suffit que $N$ soit de la forme

$$
N=c \varepsilon+\int G_{L, p} d \nu(p)
$$

où c est une constante $\geqq 0$ et où $\nu$ est une mesure positive sur $\boldsymbol{R}^{+}$telle que, quel que soit $m$ un entier $\geqq 0, \int p^{m} d \nu(p)<+\infty$.

On rappelle que $\left(G_{L, p}\right)_{p \geqq 0}$ est la résolvante associée au noyau $G_{L}$.

Démonstration. Pour tout $p>0$ et pour tout l'entier $m \geqq 0$, on a $L^{m} G_{L, p}=p^{m} G_{L, p} \leqq p^{m} G_{L, p}$ en dehors de l'origine, et donc il en résulte immédiatement que la condition est suffisante.

Montrons que la condition est nécessaire. De la même manière que dans le lemme 3 , on peut supposer que $L$ est uniformément elliptique. D'après une certaine transformation linéaire de $\boldsymbol{R}^{m}$ à lui-même, on peut supposer $L=\Delta-a$, où $a$ est une constante $\geqq 0$ d'après l'existence de $G_{L}$. D'après $L^{m} N \leqq c_{m} G_{L}$ en dehors de l'origine, il existe un noyau de convolution $N^{(m)}$ sur $\boldsymbol{R}^{n}$ tel que, quelle que soit une fonction $\varphi$ finite et continue dans $\boldsymbol{R}^{n}$ à support compact, $\int \varphi d N^{(m)}=\int_{|x|>0} \varphi(x) d\left(L^{m} N\right)(x)$. Pour tout l'entier $m \geqq 0, N^{(m)}$ est symétrique par rapport à l'origine et complètement $L$-sous-harmonique. $\quad N^{(m)}$ s'annulant à l'infini et $L N^{(m)}$ étant un laplacien généralisé symétrique sur $\boldsymbol{R}^{n}$, on obtient d'après le lemme 3 et théorème de Levy-Khinchine,

$$
\int_{|x|>0} \frac{|x|^{2}}{1+|x|^{2}} d\left(L N^{(m)}\right)(x)<+\infty .
$$

Donc, pour tout l'entier $m \geqq 1, \int d N^{(m)}<\infty$. $-\widehat{L N}$ étant définie-négative et à valeurs réelles, et pour tout l'entier $m \geqq 1$, la fonction $\hat{N}(m x)$ de $x$ étant de type positif, il existe deux constantes $c_{1} \geqq 0$ et $c_{2} \geqq 0$ telles que 


$$
-\widehat{L N}(x)=c_{1}+c_{2}|x|^{2}+\int d N^{(1)}-\widehat{N^{(1)}}(x) .
$$

D'autre part, d'après le lemme 4 , il existe uniquement une constante $c \geqq 0$ et une famille $\left(\alpha_{\sigma}\right)_{\sigma \in S_{1,0}}$ de mesures positives sur $\boldsymbol{R}^{+}$portées par $[a, \infty)$ telles que

$$
\hat{N}(r, \sigma)=c+\int \frac{1}{p+4 \pi^{2} r^{2}} d \alpha_{\sigma}(p) .
$$

Soit $\alpha_{\sigma}^{\prime}$ la mesure obtenue de $\alpha_{\sigma}$ par la translation de $-a$. On a alors

$$
\begin{aligned}
\hat{L N}(r, \sigma) & =-\left(4 \pi^{2} r^{2}+a\right) \hat{N}(r, \sigma) \\
& =-c\left(4 \pi^{2} r^{2}+a\right)+\int\left(\frac{p-a}{p+4 \pi^{2} r^{2}}-1\right) d \alpha_{\sigma}(p) \\
& =-c\left(4 \pi^{2} r^{2}+a\right)+\int\left(\frac{p}{p+a+4 \pi^{2} r^{2}}-1\right) d \alpha_{\sigma}^{\prime}(p) .
\end{aligned}
$$

Par conséquent, on a $\int d \alpha_{\rho}^{\prime}<+\infty$ et

$$
\widehat{N^{(1)}}(r, \sigma)=\int \frac{p}{p+a+4 \pi^{2} r^{2}} d \alpha_{\sigma}^{\prime}(p) \text {. }
$$

Par récurrence, on obtient que, pour tout l'entier $m \geqq 1$,

$$
\widehat{N^{(m)}}(r, \sigma)=\int \frac{p^{m}}{p+a+4 \pi^{2} r^{2}} d \alpha_{\sigma}^{\prime}(p) \text {. }
$$

Remarquons ici que $\widehat{N^{(m)}}$ est finie et continue à l'origine; alors on a, pour tout $\sigma$ de $S_{1,0}, \int \frac{p^{m}}{p+a} d \alpha_{\sigma}^{\prime}(p)<+\infty$ et $\int \frac{p^{m}}{p+a} d \alpha_{\sigma}^{\prime}(p)$ ne dépend pas de $\sigma$. Par conséquent, pour tout $t \geqq 0$, l'intégrale $\int \exp (-p t) \frac{p}{p+a} d \alpha_{\sigma}^{\prime}(p)$ est finie et ne dépend pas de $\sigma$. D'après l'injectivité de la transformation de Laplace, la restriction de $\alpha_{\sigma}^{\prime}$ dans $(0, \infty)$ ne dépend pas de $\sigma$. En utilisant encore le lemme 4, il existe une mesure $\nu^{\prime}$ sur $\boldsymbol{R}^{+}$avec $\nu^{\prime}(\{0\})=0$ et une fonction finie continue $q(\sigma) \geqq 0$ sur $S_{1,0}$ telles que

$$
\hat{N}(r, \sigma)=c+\frac{q(\sigma)}{a+4 \pi^{2} r^{2}}+\int \frac{1}{p+a+4 \pi^{2} r^{2}} d \nu^{\prime}(p) .
$$

En appliquant le théorème de Levy-Khinchine à la fonction définie-négative $-\widehat{L N}(x)$, on obtient que $q(\sigma)$ est constante. En posant $\nu=q(\sigma) \varepsilon+\nu^{\prime}$, 
on obtient $\hat{N}(x)=c+\int \frac{1}{p+a+4 \pi^{2}|x|^{2}} d \nu(p)$, d'où

$$
N=c \varepsilon+\int G_{L, p} d \nu
$$

La démonstration est ainsi complète.

Soient $L$ et $G_{L}$ les mêmes que ci-dessus. On note $\tilde{C}_{R, b}\left(G_{L}\right)$ la totalité des noyaux de convolution complètement $L$-sous-harmoniques $N$ sur $\boldsymbol{R}^{n}$ symétriques par rapport à l'origine tels que, pour tout l'entier $m \geqq 0$, il existe une constante $c_{m}>0$ vérifiant $L^{m} N \leqq c_{m} G_{L}$ en dehors de l'origine. Posons $\tilde{C}_{R}\left(G_{L}\right)=\overline{\tilde{C}_{R, b}\left(G_{L}\right)}$, où l'adhérence est au sens de la topologie vague.

CoRollaire 4. Soit $N$ un noyau de convolution sur $\boldsymbol{R}^{n}$. Alors pour que $N$ appartienne à $\tilde{C}_{R}\left(G_{L}\right)$, il faut et il suffit que $N$ soit de la forme

$$
N=c \varepsilon+\int G_{L, p} d \nu(p)
$$

où c est une constante $\geqq 0$ et $\nu \in M^{+}\left(\boldsymbol{R}^{+}\right)$, et tout l'élément de $\tilde{C}_{R}\left(G_{L}\right)$ est un noyau de convolution de Dirichlet sur $\boldsymbol{R}^{n}$ ou bien 0 .

Démonstration. On connaît déjà que si, pour $c \in R^{+}$et $\nu \in M^{+}\left(R^{+}\right)$, $N=c \varepsilon+\int G_{L, p} d \nu(p)$, alors $N$ est un noyau de convolution de Dirichlet sur $\boldsymbol{R}^{n}$ ou bien 0 (cf. par exemple, [5]), et donc il suffit de voir la première partie. La condition est évidemment suffisante, et on montrera seulement que la condition est nécessaire. Voyons d'abord que tout l'élément $N$ de $\tilde{C}_{R}\left(G_{L}\right)$ s'annule à l'infini. On choisit une suite $\left(N_{m}\right)_{m=1}^{\infty}$ de $\tilde{C}_{R, b}\left(G_{L}\right)$ qui converge vaguement vers $N$ avec $m \rightarrow \infty$. Soit $f$ une fonction $\geqq 0$, finie et continue dans $\boldsymbol{R}^{n}$ à support compact; alors il existe une fonction finie et continue $g \geqq 0$ dans $\boldsymbol{R}^{n}$ à support compact telle que $N * f<G_{L} * g$ sur $\operatorname{supp}(f)$. La suite $\left(N_{m} * f\right)_{m=1}^{\infty}$ converge uniformément vers $N * f$ sur tout compact, et donc il existe un entier $m_{0} \geqq 1$ tel que, quel que soit $m \geqq m_{0}, N_{m} * f \leqq G_{L} * g$ sur $\operatorname{supp}(f) . \quad N_{m} * f$ étant $L$ sous-harmonique en dehors de $\operatorname{supp}(f)$ et ayant $\lim _{|x| \rightarrow \infty} N_{m} * f(x)=0$, on obtient $N_{m} * f \leqq G_{L} * g$ sur $\boldsymbol{R}^{n}$. En faisant $m \rightarrow \infty$, on arrive à $N * f$ $\leqq G_{L} * g$ sur $\boldsymbol{R}^{n}$, d'où $N$ s'annule à l'infini. De la même manière que dans le lemme 3 , on peut supposer $L=\Delta-a$, où $a \in \boldsymbol{R}^{+}$. Alors tout l'élément $N$ de $\tilde{C}_{R}(G)$ est invariant par rotations et pour tout l'entier $m \geqq 0, L^{m} N$ 
est conditionnellement positive. D'après le lemme 4 , il existe une mesure positive $\nu^{\prime}$ sur $R^{+}$portée par $[a, \infty)$ et une constante $c \geqq 0$ telle que $\hat{N}(x)$ $=c+\int \frac{1}{p+4 \pi^{2}|x|^{2}} d \nu^{\prime}(p)$. Soit $\nu$ la mesure obtenue de $\nu^{\prime}$ par la translation de $-a$; alors on a

$$
N=c \varepsilon+\int G_{L, p} d \nu(p)
$$

d'où le corollaire 4.

Nous ne connaissons pas maintenant si $\tilde{C}_{R}\left(G_{L}\right)$ est égal à la totalité des noyaux de convolution complètement $L$-sous-harmoniques dans $\boldsymbol{R}^{n}$ symétriques par rapport à l'origine et s'annulant à l'infini.

D'après le corollaire 4 et une proposition générale obtenue dans [6], on obtient le corollaire suivant:

CoRollaire 5. Soient $L$ et $G_{L}$ les mêmes que ci-dessus. Alors $\tilde{C}_{R}\left(G_{L}\right)$ est un cône convexe vaguement fermé de vertex $0, \tilde{C}_{R}\left(G_{L}\right) \ni G_{L}$ et, pour tout l'élément $N \neq 0$ de $\tilde{C}_{R}\left(G_{L}\right)$, il existe un autre élément $N^{\prime} \neq 0$ de $\tilde{C}_{R}\left(G_{L}\right)$, et un seul tel que $N * N^{\prime}=G_{L}$.

On notera, pour un entier $m \geqq 1,\left(\boldsymbol{R}^{m}\right)^{+}=\left\{\tilde{p}=\left(p_{1}, \cdots, p_{m}\right) ; p_{i} \in \boldsymbol{R}^{+}\right.$ $(i=1,2, \cdots, m)\}$. On supposera toujours que $L$ et $G_{L}$ sont les mêmes que ci-dessus. Soient $N$ un noyau de convolution sur $\boldsymbol{R}^{n}$ et $\left(N_{\tilde{p}}\right)_{\tilde{p} \in \operatorname{m\geqq 1}_{m}\left(\boldsymbol{R}^{m}\right)+}$ une famille de noyaux de convolution sur $\boldsymbol{R}^{n}$. On dira que cette famille sera une classe divisible associée au noyau $N$ relativement au noyau $G_{L}$ si $N_{0}=N,\left(N_{p}\right)_{p \geqq 0}$ est la résolvante associée au noyau $N$ et si, pour tout l'entier $m \geqq 1, N_{\left(p_{1}, \cdots, p_{m}\right)} * N_{\left(p_{1}, \cdots, p_{m}, 0\right)}=G_{L}$ et $\left(N_{\left(p_{1}, \cdots, p_{m}, p\right)}\right)_{p \geqq 0}$ est la résolvante associée au noyau $N_{\left(p_{1}, \cdots, p_{m}\right)}$. Il est évident que pour un noyau de convolution $N$ sur $\boldsymbol{R}^{n}$, une classe divisible associée au noyau $N$ relativement au noyau $G_{L}$ est unique lorsqu'elle existe.

Rappelons encore la définition d'un cône convexe divisible $C_{R}\left(G_{L}\right)$ relatif au noyau $G_{L}$. Cela est, par définition, un cône convexe vaguement fermé de vertex 0 formé par de noyaux de convolution sur $\boldsymbol{R}^{n}$ et vérifiant les deux conditions suivantes:

(a) $C_{R}\left(G_{L}\right)-\{0\}$ est formé par de noyau de convolution de Hunt sur $\boldsymbol{R}^{n}$.

(b) $G_{L} \in C_{R}\left(G_{L}\right)$ et pour tout $N \neq 0$ de $C_{R}\left(G_{L}\right)$, le noyau de convolution dual $N^{\prime}$ de $N$ relativement au noyau $G_{L}$ appartient à $C_{R}\left(G_{L}\right)$. 
Lorsque, pour un noyau de convolution $N$ sur $\boldsymbol{R}^{n}$, il existe un seul noyau de convolution $N^{\prime}$ sur $R^{n}$ vérifiant $N * N^{\prime}=G_{L}, N^{\prime}$ s'appelle le noyau de convolution dual de $N$ relativement au noyau $G_{L}$.

Remarque 3. Soient $L$ et $G_{L}$ les mêmes que ci-dessus, et $C_{R}\left(G_{L}\right)$ un cône convexe divisible relatif au noyau $G_{L}$. Alors, pour tout $N \neq 0$ de $C_{R}\left(G_{L}\right)$, il existe la classe divisible associée au noyau $N$ relativement au noyau $G_{L}$.

En effet, il est déjà connu que, pour un noyau de convolution $N$ appartenant à $C_{R}\left(G_{L}\right)$, la résolvante associée au noyau $N$ est aussi contenue dans $C_{R}\left(G_{L}\right)$ (cf. [7]). En utilisant la condition (b) et par récurrence, on obtient facilement la remarque 3 .

Remarque 4. Soit $N$ un noyau de convolution sur $\boldsymbol{R}^{n}$ et supposons qu'il existe la classe divisible $\left(N_{\tilde{p}}\right)_{\tilde{p} \in \underset{m \geq 1}{\cup}\left(\boldsymbol{R}^{m}\right)+}$ associée au noyau $N$ relativement au noyau $G_{L}$. Alors, pour tout l'entier $m \geqq 1$ et pout $\tilde{p}=\left(p_{1}, \cdots\right.$, $\left.p_{m}\right)$ de $\left(\boldsymbol{R}^{m}\right)^{+}$, en posant, pour $\tilde{q}=\left(q_{1}, \cdots, q_{k}\right)$ de $\bigcup_{k \geqq 1}\left(\boldsymbol{R}^{k}\right)^{+}$,

$$
N_{\tilde{q}}^{(\tilde{p})}=N_{\left(p_{1}, \cdots, p_{m-1}, p_{m+q_{1}}, q_{2}, \cdots, q_{k}\right)},
$$

$\left(N_{\tilde{q}}^{(\tilde{p})}\right)_{\tilde{q} \in \bigcup_{k \geqq 1}^{\cup}\left(\boldsymbol{R}^{k}\right)+}$ est la classe divisible associée au noyau $N_{\tilde{p}}$ relativement au noyau $G_{L}$.

Soient $N$ un noyau de convolution sur $\boldsymbol{R}^{n}$ et $\left(N_{\tilde{p}}\right)_{\tilde{p} \in \underset{m \geqq 1}{\cup}\left(\boldsymbol{R}^{m}\right)+}$ la classe divisible associée au noyau $N$ relativement au noyau $G_{L}$. Soit $\left(p_{i}\right)_{i=1}^{m}$ une famille de $m$ nombres $>0$. On dira qu'un noyau de convolution $M$ sur $\boldsymbol{R}^{n}$ sera un noyau de convolution divisible défini par $N$ et $\left(p_{i}\right)_{i=1}^{m}$ s'il existe une famille $\left(c_{i}\right)_{i=1}^{m-1}$ de constantes $>0$ telle que

$$
M=p_{1} \cdots p_{m} N * N_{\left(0, p_{1}\right)} * \cdots * N_{\left(0, p_{1}, \cdots, p_{m}\right)}+\sum_{i=1}^{m-1} c_{i} N_{\left(0, p_{1}, \cdots, p_{i}\right)} * \cdots * N_{\left(0, p_{1}, \cdots, p_{m}\right)}
$$

sur $\boldsymbol{R}^{n}$.

Proposition 8. Soient $m$ un entier $\geqq 1$ et $\left(p_{i}\right)_{i-1}^{m}$ une famille de nombres $>0$. Alors $i$ existe une famille $\left(c_{i}\right)_{i=1}^{m-1}$ de constantes $>0$ dépendant seulement $\left(p_{i}\right)_{i=1}^{m}$, et une seule telle que, pour tout $G_{L}$, tout le noyau de convolution $N_{0}$ sur $\boldsymbol{R}^{n}$ tel qu'il existe la classe divisible $\left(N_{\tilde{p}}\right)_{\tilde{p} \in \underset{m \geqq 1}{\cup}\left(\boldsymbol{R}^{m}\right)+}$ associée au noyau $N_{0}$ relativement au noyau $G_{L}$ et pour tout l'entier $k$ avec $0 \leqq k \leqq m-1, L^{k} M / p_{1}^{2} \cdots p_{k}^{2}$ soit égal à un noyau de convolution divisible défini par $N_{\left(0, p_{1}, \cdots, p_{k}\right)}$ et $\left(p_{i}\right)_{i=k+1}^{n}$ en dehors de l'origine, où 


$$
M=p_{1} \cdots p_{m} N_{0} * N_{\left(0, p_{1}\right)} * \cdots * N_{\left(0, p_{1}, \cdots, p_{m}\right)}+\sum_{i=1}^{m-1} c_{i} N_{\left(0, p_{1}, \cdots, p_{i}\right)} * \cdots * N_{\left(0, p_{1}, \cdots, p_{m}\right)}
$$

et dans le cas où $k=0$, on suppose que $p_{1}^{2} \cdots p_{k}^{2}$ et $\left(0, p_{1}, \cdots, p_{k}\right)$ sont respectivement égals à 1 et 0 .

Montrons cette proposition par récurrence pour $m$. Il est évident que notre énoncé a lieu dans le cas où $m=1$. Supposons que dans le cas où $m=s(\geqq 1)$, notre énoncé a lieu pour tout la famille de $s$ nombres $>0$. Soit $\left(p_{i}\right)_{i=1}^{s+1}$ une famille de nombres $>0$. On désigne par $\left(c_{i}^{\prime}\right)_{i=1}^{s-1}$ la unique famille de constantes $>0$ obtenue pour $\left(p_{i}\right)_{i=2}^{s+1}$. Posons $M_{s}=p_{2} \cdots p_{s+1} N_{\left(0, p_{1}\right)} * \cdots * N_{\left(0, p_{1}, \cdots, p_{s+1}\right)}+\sum_{i=1}^{s-1} c_{i}^{\prime} N_{\left(0, p_{1}, \cdots, p_{i+1}\right)} * \cdots * N_{\left(0, p_{1}, \cdots, p_{s+1}\right)}$.

Alors $M_{s}$ un noyau de convolution divisible défini par $N_{\left(0, p_{1}\right)}$ et $\left(p_{i}\right)_{i=2}^{s+1}$. Pour tout l'entier $i$ avec $1 \leqq i \leqq s$, on a $N_{\left(0, p_{1}, \cdots, p_{i-1}\right)} * N_{\left(0, p_{1}, \cdots, p_{i-1}, 0\right)}=G_{L}$. Donc $N_{\left(0, p_{1}, \cdots, p_{i-1}, 0\right)}$ s'annule à l'infini, et par suite on a $p_{i} \int d N_{\left(0, p_{1}, \cdots, p_{i}\right)} \leqq 1$ (cf. [6]). Par conséquent $\int d M_{s}<+\infty$, et par suite

$$
M_{s+1}=\sum_{i=1}^{s-1} \frac{c_{i} p_{1}^{2}}{p_{i+1} \cdots p_{s+1}} G_{L}+p_{1} G_{L} *\left(\varepsilon-p_{1} M_{s}\right)
$$

a un sens. Ayant, pour tout l'entier $i$ avec $1 \leqq i \leqq s+1$,

$$
\begin{aligned}
G_{L} *(\varepsilon- & \left.p_{i} \cdots p_{s+1} N_{\left(0, p_{1}, \cdots, p_{i}\right)} * \cdots * N_{\left(0, p_{1}, \cdots, p_{s+1}\right)}\right) \\
= & \sum_{k=0}^{s-i} p_{i+k+1} \cdots p_{s+1}\left(G_{L} *\left(\varepsilon-p_{i+k} N_{\left(0, p_{1}, \cdots, p_{i+k}\right)}\right)\right) * N_{\left(0, p_{1}, \cdots, p_{i+k+1}\right)} \\
& * \cdots * N_{\left(0, p_{1}, \cdots, p_{s+1}\right)}+G_{L} *\left(\varepsilon-p_{s+1} N_{\left(0, p_{1}, \cdots, p_{s+1}\right)}\right) \\
= & \sum_{k=0}^{s-i} p_{i+k+1} \cdots p_{s+1} N_{\left(0, p_{1}, \cdots, p_{i+k-1}\right)} * \cdots * N_{\left(0, p_{1}, \cdots, p_{s+1}\right)}+N_{\left(0, p_{1}, \cdots, p_{s}\right)} \\
& * N_{\left(0, p_{1}, \cdots, p_{s+1}\right)}
\end{aligned}
$$

on obtient qu'il existe une famille $\left(c_{i}\right)_{i=1}^{s}$ de constantes $>0$ dépendant seulement de $\left(p_{i}\right)_{i=1}^{s+1}$ telle que

$$
\begin{aligned}
M_{s+1}= & p_{1} \cdots p_{s+1} N_{0} * N_{\left(0, p_{1}\right)} * \cdots * N_{\left(0, p_{1}, \cdots, p_{s+1}\right)} \\
& +\sum_{i=1}^{s} c_{i} N_{\left(0, p_{1}, \cdots, p_{i}\right)} * \cdots * N_{\left(0, p_{1}, \cdots, p_{s+1}\right)} .
\end{aligned}
$$

On a $L M_{s+1}=p_{1}^{2} M_{s}$ en dehors de l'origine. D'après notre hypothèse, pour tout l'entier $k$ avec $0 \leqq k \leqq s, L^{k} M_{s+1} / p_{1}^{2} \cdots p_{k}^{2}$ est égal à un noyau 
de convolution divisible défini par $N_{\left(0, p_{1}, \cdots, p_{k}\right)}$ et $\left(p_{i}\right)_{i=k+1}^{s+1}$ en dehors de 0 .

Voyons finalement l'unicité de $\left(c_{i}\right)_{i=1}^{s}$. Soit $\left(\tilde{c}_{i}\right)_{i=1}^{s}$ une autre famille de constantes $>0$ qui vérifie les mêmes conditions que pour $\left(c_{i}\right)_{i=1}^{s}$. On désigne par $M_{s+1}^{\prime}$ le noyau de convolution divisible défini par $N_{0}$ et $\left(p_{i}\right)_{i=1}^{s+1}$ obtenu de $\left(\tilde{c}_{i}\right)_{i=1}^{s}$. D'après notre hypothèse, on obtient que, pour tout $G_{L}$ et pour tout $N_{0}, L M_{s+1}=L M_{s+1}^{\prime}$ en dehors de l'origine. D'autre part, on obtient que, pour tout l'entier $i$ avec $0 \leqq i \leqq s$,

$$
N_{\left(0, p_{1}, \cdots, p_{i}\right)} * N_{\left(0, p_{1}, \cdots, p_{i+1}\right)}=G_{L} *\left(\varepsilon-p_{i_{+1}} N_{\left(0, p_{1}, \cdots, p_{i+1}\right)}\right) .
$$

Donc il existe une constante $c$ telle que $M_{s+1}-M_{s+1}^{\prime}=c G_{L}$, et $c$ ne dépand pas de $G_{L}$ et de $N_{0}$, car d'après le corollaire 5 , pour tout $p>0$, il existe la classe divisible associée au noyau $G_{L, p}$ relativement au noyau $G_{L}$. Par conséquent, $c=0$, et par suite $c_{i}=\tilde{c}_{i}(i=1,2, \cdots, s)$, d'après le corollaire 5 . La démonstration est ainsi complète.

Pour la famille $\left(c_{i}\right)_{i=1}^{m-1}$ obtenue ci-dessus, on note $c_{j}=c_{j}\left(\left(p_{i}\right)_{i=1}^{m}\right)$ $(1 \leqq j \leqq m-1)$. Le noyau de convolution $M$ dans la présente proposition s'appellera le noyau de convolution divisible d'ordre $m$ défini par $N_{0}$ et $\left(p_{i}\right)_{i=1}^{m}$.

Remarque 5. Soit $\left(p_{i}\right)_{i=1}^{m}$ une famille de nombres $>0$ et supposons que $G_{L}, N_{0}$ et $\left(N_{\tilde{p}}\right)_{\tilde{p} \in \underset{m \geqq 1}{\cup}\left(\boldsymbol{R}^{m}\right)+}$ sont les mêmes que ci-dessus. Pour le noyau de convolution divisible $M$ d'ordre $m$ défini par $N_{0}$ et $\left(p_{i}\right)_{i=1}^{m}$, et pour un entier $k$ avec $D \leqq k \leqq m-1, L^{k} M / p_{1}^{2} \cdots p_{k}^{2}$ est aussi égal au noyau de convolution divisible d'ordre $m-k$ défini par $N_{\left(0, p_{1}, \cdots, p_{k}\right)}$ et $\left(p_{i}\right)_{i=k+1}^{m}$ en dehors de l'origine.

Cela résulte immédiatement de la proposition 8.

LEMme 6. Soient $G_{L}, N_{0},\left(N_{\tilde{p}}\right)_{\tilde{p} \in \underset{m \geq 1}{\cup}\left(R^{m}\right)^{+}}, m$ et $\left(p_{i}\right)_{i=1}^{m}$ les mêmes que ci-dessus. Alors on a

$$
\lim _{p_{m+1} \rightarrow \infty} \sum_{j=1}^{m} \frac{c_{j}\left(\left(p_{i}\right)_{i=1}^{m+1}\right)}{p_{j} \cdots p_{m+1}}=\sum_{j=1}^{m-1} \frac{c_{j}\left(\left(p_{i}\right)_{i=1}^{m}\right)}{p_{j} \cdots p_{m}}
$$

et, pour toute la fonction continue et bornée $f$ dans $\boldsymbol{R}^{n}$,

$$
\lim _{p_{m+1} \rightarrow \infty} \int f d M_{m+1}^{(2)}=\int f d M_{m}^{(2)}
$$

où 


$$
M_{m+1}^{(2)}=\sum_{j=1}^{m} c_{j}\left(\left(p_{i}\right)_{i=1}^{m+1}\right) N_{\left(0, p_{1}, \cdots, p_{j}\right)} * \cdots * N_{\left(0, p_{1}, \cdots, p_{m+1}\right)}
$$

et

$$
M_{m}^{(2)}=\sum_{j=1}^{m-1} c_{j}\left(\left(p_{i}\right)_{i=1}^{m}\right) N_{\left(0, p_{1}, \cdots, p_{j}\right)} * \cdots * N_{\left(0, p_{1}, \cdots, p_{m}\right)} .
$$

En effet, d'après la proposition 1 , on obtient que, pour tout $\tilde{p}$ de $\bigcup_{m \geqq 1}\left(\boldsymbol{R}^{m}\right)^{+}, N_{\tilde{p}}$ est un noyau de convolution de Hunt sur $\boldsymbol{R}^{n} . N_{\tilde{p}}$ s'annulant à l'infini (cf. la démonstration de la proposition 8$)$, pour tout $\tilde{q}=\left(q_{1}, \cdots\right.$, $\left.q_{m}\right)$ de $\bigcup_{m \geqq 1}\left(\boldsymbol{R}^{m}\right)^{+}$et pour tout $p>0$, on a $p \int d N_{\left(q_{1}, \cdots, q_{m}, p\right)} \leqq 1$ et $p N_{\left(q_{1}, \cdots, q_{m}, p\right)}$ converge vaguement vers $\varepsilon$ avec $p \rightarrow \infty$.

Par conséquent on peut montrer, par récurrence pour $m-k$, que pour tout l'entier $k$ avec $0 \leqq k \leqq m-1$,

$$
\lim _{p_{m+1} \rightarrow \infty} \sum_{j=1}^{m-k} \frac{c_{j}\left(\left(p_{i}\right)_{i=k+1}^{m+1}\right)}{p_{k+j} \cdots p_{m+1}}=\sum_{j=1}^{m-k-1} \frac{c_{j}\left(\left(p_{i}\right)_{i=k+1}^{m}\right)}{p_{k_{+j}} \cdots p_{m}}
$$

toute la fonction continue et bornée $f$ dans $\boldsymbol{R}^{n}$,

$$
\lim _{p_{m+1} \rightarrow \infty} \int f d M_{\left(0, p_{1}, \cdots, p_{k}\right), m-k+1}^{(2)}=\int f d M_{\left(0, p_{1}, \cdots, p_{k}\right), m-k}^{(2)},
$$

où

$$
M_{\left(0, p_{1}, \cdots, p_{k}\right), m-k+1}^{(2)}=\sum_{j=1}^{m-k} c_{j}\left(\left(p_{i}\right)_{i=k+1}^{m+1}\right) N_{\left(0, p_{1}, \cdots, p_{k+\jmath}\right)} * \cdots * N_{\left(0, p_{1}, \cdots, p_{m+1}\right)}
$$

et

$$
M_{\left(0, p_{1}, \cdots, p_{k}\right), m-k}^{(2)}=\sum_{j=1}^{m-k-1} c_{i}\left(\left(p_{i}\right)_{i=k+1}^{m}\right) N_{\left(0, p_{1}, \cdots, p_{k+j}\right)} * \cdots * N_{\left(0, p_{1}, \cdots, p_{m}\right)} .
$$

Cela résulte facilement du fait que

$$
\begin{gathered}
M_{\left(0, p_{1}, \cdots, p_{m-1}\right), 1}^{(2)}=0, \\
M_{\left(0, p_{1}, \cdots, p_{m-1}, 2\right.}^{(2)}=p_{m} N_{\left(0, p_{1}, \cdots, p_{m}\right)} * N_{\left(0, p_{1}, \cdots, p_{m+1}\right)}
\end{gathered}
$$

et pour tout l'entier $k$ avec $1 \leqq k \leqq m-1$,

$$
\begin{aligned}
p_{k} \cdots & p_{m+1} N_{\left(0, p_{1}, \cdots, p_{k-1}\right)} * \cdots * N_{\left(0, p_{1}, \cdots, p_{m+1}\right)}+M_{\left(0, p_{1}, \cdots, p_{k-1}, m-k+2\right.}^{(2)} \\
= & \sum_{j=1}^{m-k} \frac{c_{j}\left(\left(p_{i}\right)_{i=k+1}^{m+1}\right) p_{k}^{2}}{p_{k+j} \cdots p_{m+1}} G_{L}+p_{k} G_{L} *\left(\varepsilon-p_{k} \cdots p_{m+1} N_{\left(0, p_{1}, \cdots, p_{k}\right)}\right. \\
& \left.* \cdots * N_{\left(0, p_{1}, \cdots, p_{m+1}\right)}-p_{k} M_{\left(0, p_{1}, \cdots, p_{k}\right), m-k+1}^{(2)}\right)
\end{aligned}
$$


et

$$
\begin{aligned}
p_{k} \cdots & p_{m} N_{\left(0, p_{1}, \cdots, p_{k-1}\right)} * \cdots * N_{\left(0, p_{1}, \cdots, p_{m}\right)}+M_{\left(0, p_{1}, \cdots, p_{k-1}\right), m-k+1}^{(2)} \\
= & \sum_{j=1}^{m-k-1} \frac{c_{j}\left(\left(p_{i}\right)_{i=k+1}^{m}\right) p_{k}^{2}}{p_{k+j} \cdots p_{m}} G_{L}+p_{k} G_{L} *\left(\varepsilon-p_{k} \cdots p_{m} N_{\left(0, p_{1}, \cdots, p_{k}\right)}\right. \\
& \left.* \cdots * N_{\left(0, p_{1}, \cdots, p_{m}\right)}-p_{k} M_{\left(0, p_{1}, \cdots, p_{k}\right), m-k}^{(2)}\right),
\end{aligned}
$$

d'après la démonstration de la proposition 8 .

On montrera ici notre théorème principal.

THÉORÈME. Soit L un opérateur différentiel elliptique et auto-adjoint d'ordre $\leqq 2$ sur $\boldsymbol{R}^{n}$ à coéfficients constants et supposons qu'il existe le noyau de convolution sur $\boldsymbol{R}^{n}$ s'annulant à l'infini avec $L G_{L}=-\varepsilon$. Alors un cône convexe divisible relatif au noyau $G_{L}$ formé par de noyaux de convolution symétriques par rapport à l'origine est uniquement déterminé, et cela est égal à

$$
\left\{c \varepsilon+\int G_{L, p} d \nu(p) ; c \in \boldsymbol{R}^{+} \quad \text { et } \quad \nu \in M^{+}\left(\boldsymbol{R}^{+}\right) \text {avec } \int_{1}^{\infty} \frac{1}{p} d \nu(p)<+\infty\right\} \text {. }
$$

Démonstration. Soit $C_{R}\left(G_{L}\right)$ un cône convexe divisible quelconque relatif au noyau $G_{L}$ constitué par de noyaux de convolution symétriques par rapport à l'origine. Pour la première partie du théorème, il suffit de voir $C_{R}\left(G_{L}\right)=\tilde{C}_{R}\left(G_{L}\right)$. D'après une proposition générale obtenue dans [6] et le corollaire 4 , on a $C_{R}\left(G_{L}\right) \supset \tilde{C}_{R}\left(G_{L}\right)$. Donc il suffit de montrer que $\tilde{C}_{R, b}\left(G_{L}\right)$ est dense dans $C_{R}\left(G_{L}\right)$ pour la topologie vague. Soit $N$ un noyau de convolution $\neq 0$ sur $R^{n}$ appartenant à $C_{R}\left(G_{L}\right)$. Pour $N \in \overline{\widetilde{C}_{R, b}\left(G_{L}\right)}$, il suffit de supposer $\int d N<+\infty$, car la résolvante associée au noyau $N$ est aussi contenue dans $C_{R}\left(G_{L}\right)$. D'après la remarque 3 , on désigne par $\left(N_{\tilde{p}}\right)_{\tilde{p} \in \underset{m \geqq 1}{\cup}\left(R^{m}\right)+}$ la classe divisible associée au noyau $N$ relativement au noyau $G_{L}$. Soit $\delta$ un nombre $>0$ quelconque donné. Alors il existe une suite croissante $\left(p_{m}\right)_{m=1}^{\infty}$ de nombres $>0$ telles que, en posant

$$
M_{m}^{(1)}=p_{1} \cdots p_{m} N * N_{\left(0, p_{1}\right)} * \cdots * N_{\left(0, p_{1}, \cdots, p_{m}\right)},
$$

on ait

$$
0 \leqq \hat{N}(x)-\widehat{M_{m}^{(1)}}(x) \leqq\left(1-\frac{1}{2^{m}}\right) \delta \quad \text { sur } \overline{B_{1,0}}
$$

et pour tous les entiers $k \geqq 1$ et $s \geqq 1$, 


$$
\sum_{j=1}^{s-1} \frac{c_{j}\left(\left(p_{i}\right)_{i=k+1}^{k+s}\right)}{p_{k+j} \cdots p_{k+s}} \leqq\left(1-\frac{1}{2^{s-1}}\right) \delta
$$

Rappelons le lemme 5 et que, pour tout $\tilde{q}=\left(q_{1}, \cdots, q_{m}\right), p \widehat{N_{\left(q_{1}, \cdots, q_{m}, p\right)}}$ converge uniformément vers 1 sur tout compact de $\boldsymbol{R}^{n}$; alors il est facile de voir, par récurrence, l'existence de cette suite. Posons

$$
M_{m}=M_{m}^{(1)}+M_{m}^{(2)},
$$

où

$$
M_{m}^{(2)}=\sum_{j=1}^{m-1} c_{j}\left(\left(p_{i}\right)_{i=1}^{m}\right) N_{\left(0, p_{1}, \cdots, p_{j}\right)} * \cdots * N_{\left(0, p_{1}, \cdots, p_{m}\right)} .
$$

D'après la manière d'obtenir la famille $\left(c_{j}\left(\left(p_{i}\right)_{i=1}^{m}\right)\right)_{j=1}^{m-1}$ dans la proposition 8 , on a, pour tout $m \geqq 2$,

$$
M_{m} \leqq\left(\sum_{j=1}^{m-2} \frac{c_{j}\left(\left(p_{i}\right)_{i=2}^{m}\right) p_{1}^{2}}{p_{j+1} \cdots p_{m}}+p_{1}\right) G_{L} \leqq p_{1}\left(1+p_{1}\left(1-\frac{1}{2^{m-2}}\right) \delta\right) G_{L}
$$

dans $\boldsymbol{R}^{n}$, et on a immédiatement

$$
M_{1}=p_{1} N * N_{\left(0, p_{1}\right)}=p_{1} G_{L} *\left(\varepsilon-p_{1} N_{\left(0, p_{1}\right)}\right) \leqq p_{1} G_{L}
$$

dans $R^{n}$. D'autre part, d'après la remarque 5, pour tout l'entier $k$ avec $1 \leqq k \leqq m-1$,

$$
\begin{aligned}
L^{k} M_{m}= & p_{1}^{2} \cdots p_{k}^{2}\left(p_{k+1} \cdots p_{m} N_{\left(0, p_{1}, \cdots, p_{k}\right)} * \cdots * N_{\left(0, p_{1}, \cdots, p_{m}\right)}\right. \\
& \left.+\sum_{j=1}^{m-k-1} c_{j}\left(\left(p_{i}\right)_{i=k+1}^{m}\right) N_{\left(0, p_{1}, \cdots, p_{k+j}\right)} * \cdots * N_{\left(0, p_{1}, \cdots, p_{m}\right)}\right)
\end{aligned}
$$

en dehors de l'origine. De la même manière que pour le noyau de convolution $M_{m}$, on a, pour tout l'entier $k$ avec $1 \leqq k \leqq m-2$,

$$
L^{k} M_{m} \leqq p_{1}^{2} \cdots p_{k}^{2} p_{k+1}\left(1+p_{k+1}\left(1-\frac{1}{2^{m-k-2}}\right) \delta\right) G_{L}
$$

et

$$
L^{m-1} M_{m} \leqq p_{1}^{2} \cdots p_{m-1}^{2} p_{m} G_{L}
$$

en dehors de l'origine. Ayant $\int d N<+\infty$, et $N-M_{m}^{(1)}$ et $M_{m}^{(1)}-M_{m+1}^{(1)}$ étant de type positif, on obtient que $\left(M_{m}^{(1)}\right)_{m=1}^{\infty}$ converge vaguement vers un noyau de convolution $M_{\dot{\delta}}^{(1)}$ sur $\boldsymbol{R}^{n}$ avec $m \rightarrow \infty$ et que 


$$
\lim _{m \rightarrow \infty} \widehat{M_{m}^{(1)}}(x)=\widehat{M_{\tilde{\delta}}^{(1)}}(x) \quad \text { sur } \quad R^{n}
$$

D'autre part, on a

$$
\int d M_{m}^{(2)} \leqq \sum_{j=1}^{m-1} \frac{c_{j}\left(\left(p_{i}\right)_{i=1}^{m}\right)}{p_{j} \cdots p_{m}}<\delta
$$

Donc on peut supposer que $\left(M_{m}^{(2)}\right)_{m=1}^{\infty}$ converge vaguement vers un noyau de convolution $M_{\delta}^{(2)}$ avec $m \rightarrow \infty$. Posons $\tilde{N}_{\delta}=M_{\delta}^{(1)}+M_{\delta}^{(2)}$; alors on a

$$
0 \leqq \tilde{N}_{\delta} \leqq\left(1+p_{1} \delta\right) p_{1} G_{L} \quad \text { dans } \quad R^{n}
$$

et, pour tout l'entier $k \geqq 1$,

$$
0 \leqq L^{k} \tilde{N}_{\delta} \leqq\left(1+p_{k+1} \delta\right) p_{1}^{2} \cdots p_{k}^{2} p_{k+1} G_{L}
$$

en dehors de l'origine. Il existe un noyau de convolution $M_{\dot{\delta}}^{\prime}$ sur $\boldsymbol{R}^{n}$ symétrique par rapport à l'origine avec $\int d M_{\delta}^{\prime} \leqq 1$ tel que $M_{\dot{\delta}}^{(1)}=N * M_{\delta}^{\prime}$. D’après les inégalités

$$
0 \leqq \hat{N}(x)-\widehat{M_{\delta}^{(1)}}(x) \leqq \delta \quad \text { sur } \quad \overline{B_{1,0}}
$$

et $\int d N<+\infty$, on obtient que $M_{\delta}^{(1)}$ converge vaguement vers $N$ avec $\delta \rightarrow 0$. Ayant $\int d M_{\delta}^{(2)} \leqq \delta$, on obtient que $\tilde{N}_{\delta}$ converge vaguement vers $N$ avec $\delta \rightarrow 0$, d'où $\tilde{C}_{R, b}\left(G_{L}\right)$ est dense dans $C_{R}\left(G_{L}\right)$.

Montrons finalement que, pour une mesure positive $\nu$ sur $\boldsymbol{R}^{+}$, $\int G_{L, p} d \nu(p)$ définit un noyau de convolution sur $\boldsymbol{R}^{n}$ si et seulement si $\int_{1}^{\infty} \frac{1}{p} d \nu(p)<+\infty$. En effet, pour une fonction finie et continue $f \geqq 0$ dans $\boldsymbol{R}^{n}$ à support compact telle que $f(0)>0$,

$$
0<\inf _{p \geqq 1} p \int f d G_{L, p} \leqq \sup _{p \geqq 1} p \int f d G_{L, p} \leqq \max _{x \in R^{n}} f(x),
$$

car $G_{L, p}$ est de type positif et $\left(p G_{L, p}\right)_{p>0}$ converge vaguement vers $\varepsilon$ avec $p \rightarrow \infty$. Donc on a l'équivalence

$$
\iint f d G_{L, p} d \nu(p)<+\infty \Leftrightarrow \int_{1}^{\infty} \frac{1}{p} d \nu(p)<+\infty .
$$

La démonstration est ainsi complète. 
Remarque 6. Soient $L$ et $G_{L}$ les mêmes que ci-dessus. De la même manière que ci-dessus, on peut montrer que, pour tout le cône convexe divisible $C_{R}\left(G_{L}\right)$ relatif au noyau $G_{L}$ et pour tout le noyau de convolution $N$ sur $R^{n}$ appartenant à $C_{R}\left(G_{L}\right), N+\check{N}$ appartient à $C_{R}\left(G_{L}\right)$ et cela est de la forme

$$
N+\check{N}=c \varepsilon+\int G_{L, p} d \nu(p)
$$

où $c \in R^{+}$et $\nu \in M^{+}\left(R^{+}\right)$avec $\int_{1}^{\infty} \frac{1}{p} d \nu(p)<+\infty$.

On note ici $\check{N}$ le noyau de convolution symétrisant avec $N$ par rapport à l'origine.

\section{RÉFÉRENCES}

[1] A. Beurling et J. Deny: Dirichlet spaces, Proc. Nat. Acad. Sc. U.S.A., 45 (1959), p. 208-215.

[2] G. Choquet et J. Deny: Aspects linéaires de la théorie du potentiel, Noyaux de composition satisfaisant au principe du balayage sur tout ouvert, C. R. Acad. Sc. Paris, 250 (1960), p. 4260-4262.

[ 3 ] C. S. Herz: Analyse harmonique à plusieurs variables, Sém. Math. d'Orsay, $1965 / 66$.

[4] M. Itô: Sur les principes divers du maximum et le type positif, Nagoya Math. J., 44 (1971), 133-164.

[5] _ - Sur la famille sous-ordonnée au noyau de convolution de Hunt II, Nagoya Math. J., 53 (1974), p. 115-126.

[6] —-: Une caractérisation du principe de domination pour les noyaux de convolution, Nagoya Math. J., à paraître.

[ 7 ] — : Sur les cônes convexes de Riesz et les noyaux de convolution complètement sous-harmoniques, Nagoya Math. J., 55 (1974), p. 111-144.

[ 8 ] D. Widder: The Laplace transform, Princeton Univ. Press, Princeton, 1948.

Université de Nagoya 